\title{
ASPECTOS HIDROLÓGICOS E HIDRÁULICOS DEL ESTUDIO DE AMENAZA POR FENÓMENOS DE REMOCIÓN EN MASA EN LA QUEBRADA EL COFRE
}

\author{
HYDROLOGIC AND HYDRAULIC ASPECTS OF THE STUDY OF THREAT BY \\ MASS REMOVAL IN EL COFRE CREEK
}

\author{
Pedro León García Reinoso ${ }^{1}$ \\ Elkin Aníbal Monsalve Durango ${ }^{1}$, Gabriel Lozano Sandoval ${ }^{1}$ \\ ${ }_{1}^{1}$ Profesores programa ingenieria civil. Facultad de Ingenieria Universidad del Quindío
}

Fecha de recibido: Febrero 3 de 2010

Fecha de aceptado: Junio 9 de 2010

Correspondencia: Programa de Ingeniería Civil, Universidad del Quindío Av. Bolívar calle 12 norte Armenia Quindío. Correo electrónico: pedrogarcia@uniquindio.edu.co

\section{RESUMEN}

En el Quindío los días 24, 25 y 26 de diciembre de 1999 ocurrieron avenidas torrenciales que afectaron al corregimiento de La Virginia. Estos eventos se asociaron con la inestabilidad de las laderas inducida por el sismo de enero de 1999 y las fuertes Iluvias de diciembre. En los últimos diez años el fenómeno se ha documentado lo suficiente como para proponer acciones de intervención sustentadas en el análisis detallado de las variables que allí intervienen. La Universidad del Quindío, a través del Centro de Extensión de la Facultad de Ingeniería y con el Apoyo de los Grupos de Investigación CIDERA y QUIMBAYA, se propusieron analizar el fenómeno integrando las condiciones ambientales, geológicas, sísmicas, geotécnicas, hidrológicas e hidráulicas, con el propósito de definir los impactos que generaría la ocurrencia de un fenómeno de remoción en masa al corregimiento de La Virginia. El análisis se realizó con ArcGIS 9.3 y FLO-2D, para generar mapas de riesgo y amenaza por avalancha, respectivamente.

Palabras Clave: Hidrología. Hidráulica. Amenaza.

\section{ABSTRACT}

In Quindio, on December 24, 25, and 26 in 1999 there were some mud flows that affected the village La Virginia. These events were associated with the hillsides instability caused by the earthquake on January of 1999 and several rains on December. In the last ten years the phenomenon has been documented well enough to propose intervention actions supported in the detailed analysis of the variables that get involved there. University of Quindio, through Extension Office of Faculty of Engineering and with support of research groups CIDERA and QUIMBAYA, decided to analyze this phenomenon integrating environmental, geological, seismic, geotechnical, hydrologic and hydraulic conditions to define the impact that a phenomenon of mass removal would cause to La Virginia. The analysis was carried out with ArCGIS 9.3 and FLO-2D to generate risk and mud flood hazard maps, respectively.

Key words: Hydrology, Hydraulics, Hazard.

\section{INTRODUCCIÓN}

En el Quindío los días 24, 25 y 26 de diciembre ocurrieron avenidas torrenciales que afectaron al corregimiento de La Virginia y represaron el Río Santo Domingo. La intensidad de esos eventos se asoció con la inestabilidad de las laderas inducida por el sismo de enero de 1999 y las fuertes lluvias de diciembre (1). Los flujos destruyeron 10 viviendas, 4 puentes, el acueducto de La Virginia (2); fueron afectadas 60 viviendas y el colegio Jesús María Morales; 450 familias fueron ubicadas en el campamento temporal emplazado en la Finca Llanitos (3). En trabajo de campo adelantado por Aguilar y Mendoza (1) se obtuvieron evidencias de grietas en las laderas y cerca a los cauces de las quebradas a partir de las cuales, y por efecto de las lluvias, ocurrieron deslizamientos que, a su vez, indujeron las avenidas torrenciales del 24 y 25 de diciembre. Relata Aguilar y Mendoza (1), "que los pobladores informaron que el 24 de diciembre, entre las 3:00 p.m. y las 4:00 p.m., hubo una granizada que alcanzó una altura de $5 \mathrm{~cm}$. El granizo permaneció en las laderas y se notaba todavía el 25 de diciembre. En la madrugada del 25 de diciembre ocurrió la primera avenida, y a las once de la noche del mismo día bajó una segunda avenida mucho más fuerte, que causó muchas de las averías. 'La avenida bajo en 5 pulsos, la tierra cimbraba y se oía un ruido muy fuerte, la avenida cubrió uno de los puentes del corregimiento' afirmó un residente".

El fenómeno natural que se presenta en la Quebrada el Cofre, corregimiento de la Virginia, municipio de Calarcá, ha suscitado una serie de comentarios, que pasan de lo 
empírico a lo teórico generalizando comportamientos sin considerar la particularidad de las condiciones que allí se presentan, e incluso desconociendo los resultados de estudios previos que han sido adelantados con el apoyo de la Corporación Autónoma Regional del Quindío. Como acción de intervención en el mediano plazo (4) se generó un programa para la "Participación en la recuperación ambiental en zonas de alto riesgo reubicadas por los municipios - Mitigación en 24 sectores del Departamento". Se ejecutaron 11 obras para la mitigación de riesgos naturales por inestabilidad de taludes. En Calarcá se adelantaron obras de limpieza sobre la quebrada El Cofre.
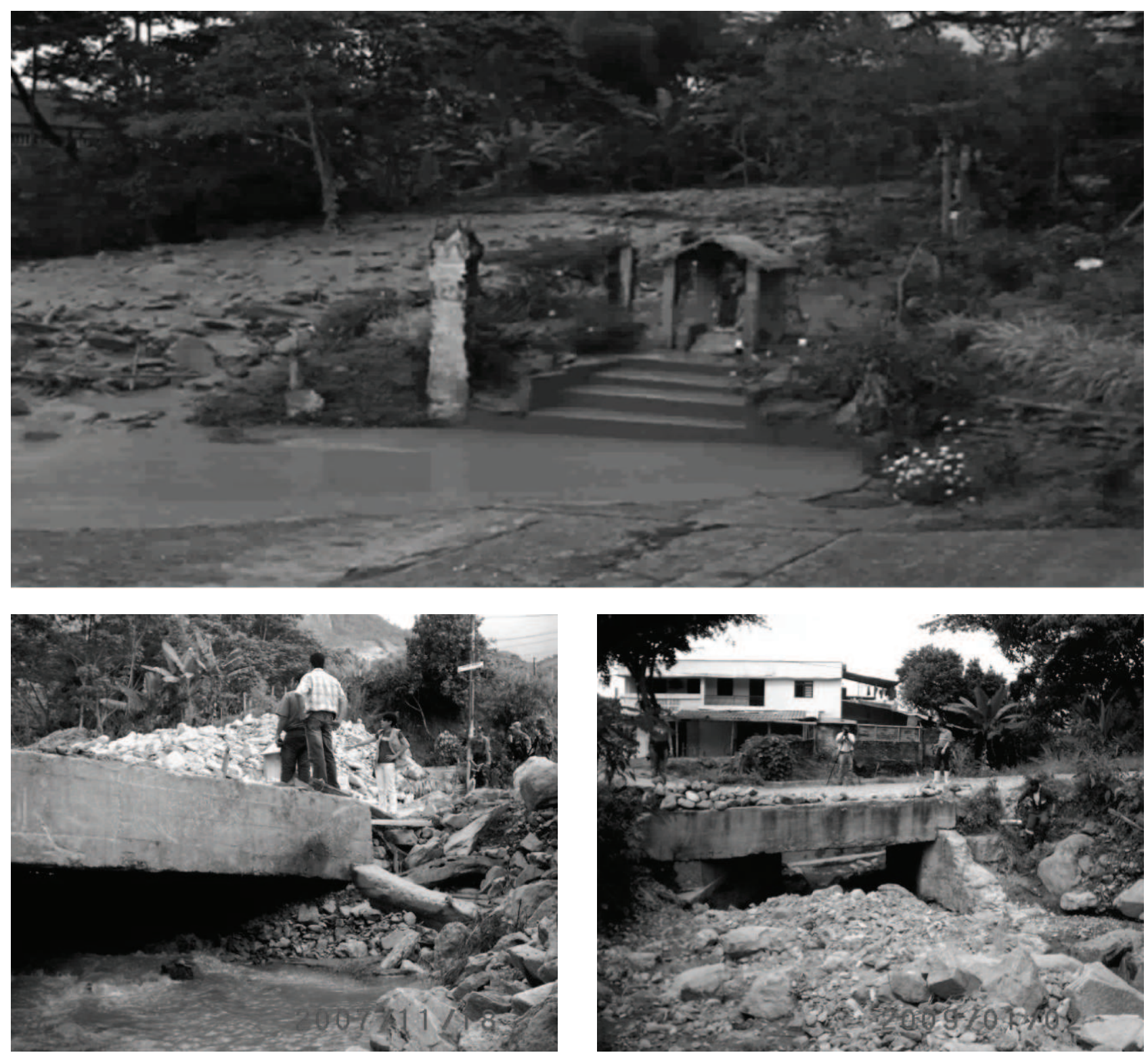

Fotografía 1. Registro gráfico de flujos generados en La Quebrada El Cofre

La evolución que ha mostrado el cauce muestra como las condiciones geométricas se han alterado dramáticamente en cortos periodos de tiempo, generando incisiones que aceleran procesos de inestabilidad existentes en los taludes que confinan lateralmente el cuerpo de agua (Fotografía 1). Este estudio pretende analizar el fenómeno con una visión holística, integrando la particularidad que hay en cuanto a las condiciones ambientales, geológicas, sísmicas, hidrológicas, geotécnicas, hidrológicas e hidráulicas, definiendo los impactos que generaría el fenómeno al corregimiento de la Virginia.

Los antecedentes dan cuenta de la inestabilidad del sector, y aunque existe una afectación directa sobre los habitantes, la población aun mantiene una posición pasiva hacía el 
fenómeno natural. En la prensa se pueden rastrear hechos verdaderamente alarmantes que no inquietan a la comunidad.

El 22 de febrero de 2008 (5), esta noticia era presentada a nivel nacional: "La alerta roja fue declarada en el corregimiento La Virginia, municipio de Calarcá, tras el represamiento de la quebrada El Cofre y la llegada al pueblo de pequeñas avalanchas de lodo y piedra, provenientes del cerro Peñas Blancas. Una de ellas fue la presentada en la tarde del miércoles, que colmató el puente sobre la quebrada en el casco urbano, dejando incomunicada una parte de la población. Quince familias fueron evacuadas de la zona aledaña al Cofre, mientras comisiones de vecinos y expertos del Comité de Atención y prevención de desastres vigilan desde varios sitios del cerro el proceso de represamiento. Cerca de veinte derrumbes se han presentado en las últimas horas en el cerro de Peñas Blancas y sobre la quebrada el Cofre, lo que ha provocado represamientos en varios sitios del cauce. El Comité de Atención y Prevención de desastres del Quindío declaró la alerta roja por posibles avalanchas de lodo y piedra sobre la población. Maquinaria y equipo del gobierno departamental iniciarán esta mañana labores para levantar el sedimento que dejó la avalancha de el miércoles a las tres y treinta de la tarde".

La evolución que mostró el fenómeno entre 1999 y 2009, ha permitido una documentación detallada, e incluso proponer acciones de intervención que están sustentadas en la observación, más no en el análisis detallado de las variables que allí intervienen. La Universidad del Quindío, a través del Centro de Extensión de la Facultad de Ingeniería y con el Apoyo de los Grupos de Investigación CIDERA y QUIMBAYA, se propusieron analizar el fenómeno con una visión holística, integrando la particularidad que hay en cuanto a las condiciones ambientales, geológicas, sísmicas, geotécnicas, hidrológicas e hidráulicas, con el propósito de definir los impactos que generaría la ocurrencia de un fenómeno de remoción en masa al corregimiento de La Virginia.

De esta forma, se desarrollaron estudios topográficos detallados que sirvieron para integrar los aspectos hidrológicos, hidráulicos, geotécnicos y geológicos con el propósito de evaluar la amenaza a la que está sometido el Corregimiento de La Virginia. En este artículo se presentan los resultados de los aspectos hidrológicos e hidráulicos. Dentro de las especialidades de la Ingeniería, uno de los primeros aspectos a evaluar es el régimen de caudales, que para el caso de la Qda. El Cofre reviste particular importancia, pues se presenta un flujo de escombros que está relacionado con fenómenos geológicos y geotécnicos. Desde el punto de vista hidráulico, los resultados sirvieron para aportar elementos técnicos que orientaran la solución que se debe dar al paso vehicular que pretende salvar el obstáculo interpuesto por la Qda. El Cofre.

\section{MATERIALES Y MÉTODOS}

\section{Zona de estudio}

El departamento del Quindío está situado en el centro geográfico del occidente colombiano. En su división político administrativa se integra con 12 municipios, entre los cuales se encuentra el municipio de Calarcá. El municipio cuenta, en la zona urbana con 95 barrios, mientras que en la el sector rural está conformado por 27 veredas, 2 centros poblados y 3 corregimientos: Barcelona, La Virginia y Quebradanegra. El corregimiento de La Virginia, se encuentra en el flanco occidental de la cordillera central, en una altitud media de 1703 msnm y está 6 kilómetros al sur de la cabecera municipal. Su topografía es quebrada y abrupta, con pendientes fuertes (6).

Dadas las características topográficas existe una rica diversidad de paisajes de acuerdo con los pisos bioclimáticos, las variaciones del relieve (predominantemente montañoso y ondulado) y las manifestaciones geológicas. En Calarcá, las altiplanicies abarcan aproximadamente el $73 \%$ de la superficie y son terrenos aptos para la agricultura como café, plátano, cítricos y explotación ganadera. Los suelos de colina equivalen al $7 \%$ de la superficie, en ellos se encuentran los corregimientos de La Virginia y Quebradanegra y las veredas La Paloma, El Calabazo y El Pensil. Los suelos de montaña abarcan el $20 \%$ del municipio, son aptos para la agricultura, pastos y bosques; a ellos pertenecen las áreas rurales de la parte media de la Cordillera Central, la cual está destinada a reserva forestal (7). Las quebradas El Cofre y Espartillal corren sobre las laderas del corregimiento, generando un aporte directo al caudal del río Santo Domingo, uno de los principales afluentes del río Quindío.

En el estudio hidrológico, el análisis cartográfico es de vital importancia para la determinación de la geometría propia del sitio, de las características físicas y de los accidentes topográficos presentes en la zona de la que dependerá el correcto funcionamiento del modelo hidráulico y los resultados obtenidos. La cartografía utilizada fue obtenida de la topografía generada en la Fase I, realizada en convenio con la Corporación Autónoma Regional del Quindío en el año 2008, y actualizada en mayo de 2009. En la Figura 1 se presenta la cuenca de la Quebrada El Cofre hasta la desembocadura en el río Santo Domingo. La parte alta de la cuenca tiene una cota de 2380 msnm y se encuentra en la esquina inferior derecha de la Figura 2, mientras que la desembocadura se localiza en el punto opuesto con una cota de $1400 \mathrm{msnm}$. El descenso de $980 \mathrm{~m}$ se hace en tan solo $3650 \mathrm{~m}$, que es la longitud del cauce principal.

En la Tabla 1 se muestran las principales características de la cuenca de estudio, la cual se consideró inicialmente hasta la desembocadura del Río Santo Domingo (Cuenca), llamado tramo 2. Se presenta un análisis separado hasta el paso vial sobre la quebrada en el sector del barrio Mariano Ospina Pérez, el cual se llamo tramo 1, ya que es el punto en donde se presentan represamiento por la obstrucción que impone la estructura al flujo de agua. 
La pendiente promedio de la cuenca es del 52,94\%, y en el Tramo 1 se incrementa hasta el 70,55\%. Esta misma situación se observa en la pendiente del media para el cauce, que para la cuenca es del 8,59\%, mientras que para el Tramo 1 del 10,29\%. La elevación media de la cuenca es de 1703 msnm, para el Tramo 1 es de 1838 msnm. Desde la perspectiva morfométrica, existe una marcada diferencia entre los dos puntos, hecho que se debe evidenciar en comportamientos del flujo de agua y escombros.

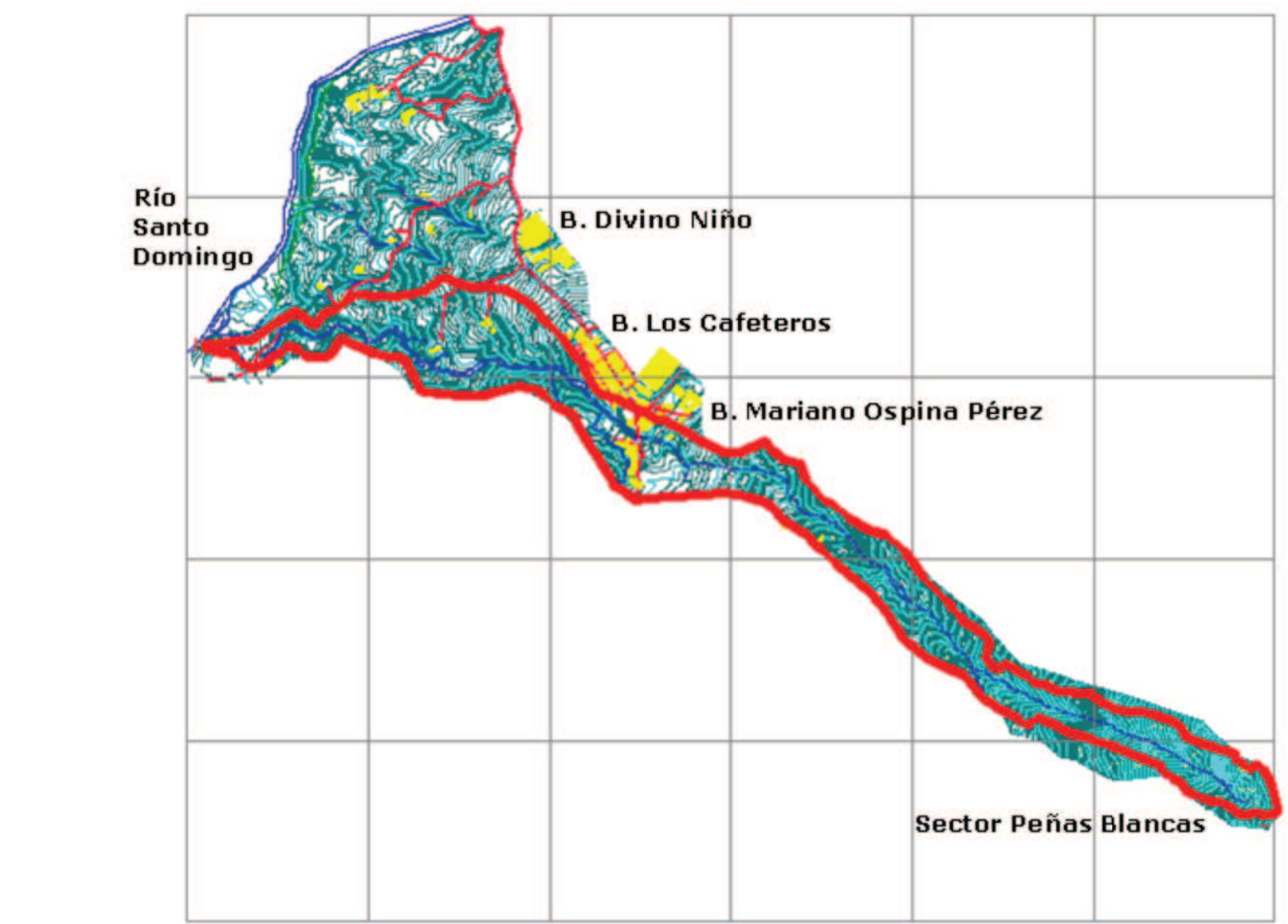

Figura 1. Cuenca de La Quebrada El Cofre (8)

Tabla 1 - Características físicas de la cuenca Quebrada El Cofre (8)

CARACTERÍSTICAS DE LA CUENCA EN ESTUDIO

PARAMETRO

\begin{tabular}{l}
\hline Longitud Cauce $(\mathrm{m})$ \\
Área $\left(\mathrm{m}^{2}\right)$ \\
Perímetro $(\mathrm{m})$ \\
Longitud Curvas de Nivel (m) \\
Coeficiente de Compacidad \\
Pendiente Media Cuenca (\%) \\
Pendiente Media Cauce (\%) \\
Altura Máxima (msnm) \\
Altura Media (msnm)
\end{tabular}

HASTA PUENTE LA VIRGINIA

HASTA DESEMBOCADURA

Altura Mínima (msn m)

$\begin{array}{cc}2.090,276 & 3.650,007 \\ 257.088,197 & 479.937,684 \\ 4.519,167 & 7.309,158 \\ 18.138,141 & 25.407,741 \\ 2,50 & 2,95 \\ 70,55 & 52,94 \\ 10,29 & 8,59 \\ 2380 & 1600 \\ 1838 & 1703 \\ 1600 & 1400\end{array}$

Rev. Invest. Univ. Quindío (20): 108 - 119. Armenia - Colombia 


\section{- 112 - Estudio de caso: amenaza por avalancha quebrada el cofre}

Para caracterizar la respuesta de la cuenca se calculo el Coeficiente de Compacidad (Kc), el cual se define como la razón entre el perímetro de la cuenca y el perímetro de una circunferencia. Por definición un valor $\mathrm{Kc}=1$ se da para cuencas imaginarias de forma circular. El grado de aproximación a la unidad indica la tendencia a concentrar grandes volúmenes. Los valores obtenidos son mayores a 1,75 , mostrando que no existe una tendencia a concentrar grandes volúmenes de agua, luego durante una tormenta se presentaría una creciente paulatina con una curva de ascenso suave.

\section{Selección de estaciones}

Se consultaron diferentes fuentes de información, de manera que se adquirió la mayor cantidad de datos y referencias posibles. La información se recopilo de documentación disponible en la Corporación Autónoma Regional del Quindío CRQ, en el Instituto de Estudios Ambientales IDEAM y Proyectos de Investigación de la Universidad del Quindío. Se analizaron registros de precipitación del Proyecto de Investigación 360 obtenidos de una estación temporal localizada en la Finca Buenavista del corregimiento de la Virginia. El registro pluviográfico va de 06/03/2008 a 03/08/2008, con registro cada 10 minutos. Aunque es un registro corto en el tiempo, su bajo nivel de agregación permite verificar valores máximos locales de intensidad. Existen registros de la CRQ en el Ecoparque Peñas Blancas de 06/11/2006 a 18/12/2006, de 10/11/2008 a $15 / 11 / 2008$ y $28 / 03 / 2009$ a 03/05/2009, la información está agregada a nivel diario y no posee continuidad en el tiempo, por ello no se utilizó en el estudio. Se analizaron Precipitaciones Máximas en 24 horas de la Estación Planadas (9) y registros de precipitación total mensual de las estaciones El Túnel, Planadas y San Rafael (10).

\section{Trabajo de campo}

La calibración del modelo hidrológico demando la selección de una estación para el aforo de caudales, con lo que se logro una correlación entre la lluvia registrada y el caudal generado, con lo que se definió el comportamiento del Número de Curva (CN). En la Figura 2 se muestra la sección elegida para el aforo de caudales.
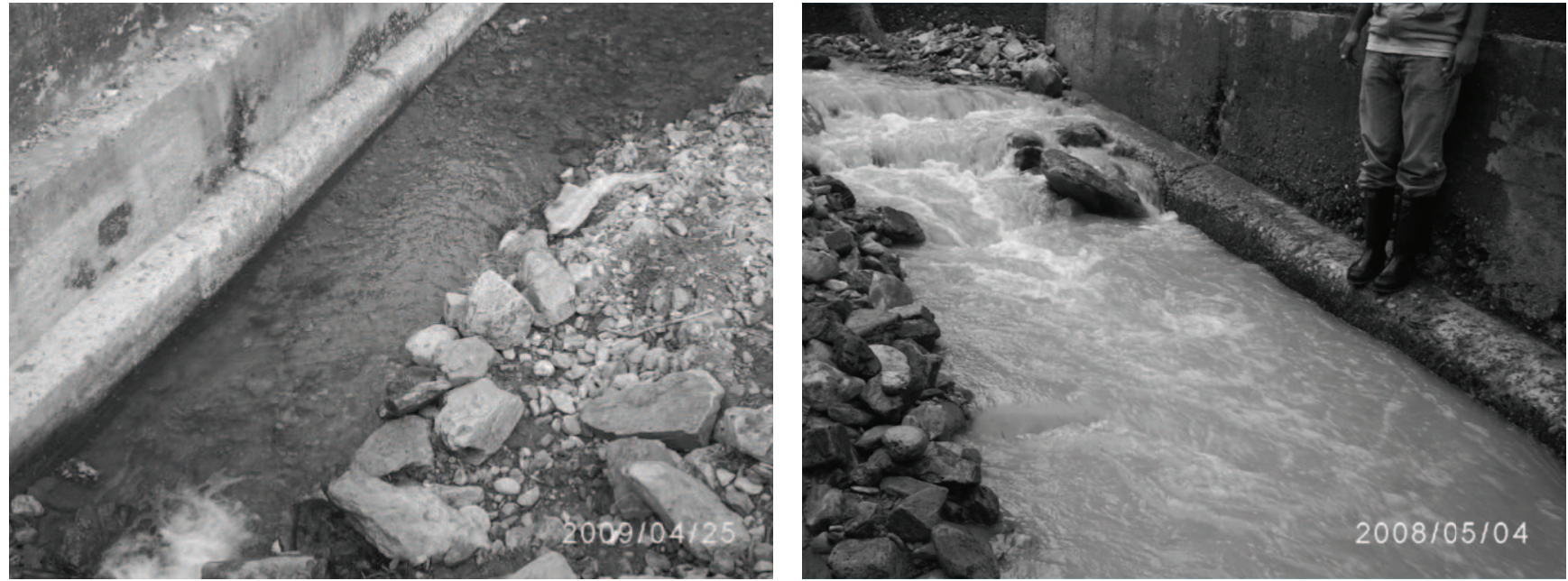

Figura 2. Estación de aforo aguas abajo paso vial de la Quebrada El Cofre ${ }^{2}$

En cuanto al estudio de sedimentos, se marcó un número considerable de rocas de diferente diámetro, las cuales estaban depositadas en la parte alta de la cuenca. La marcación se hizo con pintura resistente al agua y en colores fáciles de identificar para los diferentes diámetros. En la Figura 3 se aprecian los diferentes diámetros que fueron seleccionados para el seguimiento. En la fotografía izquierda se aprecia el fondo del cauce sin flujo de agua y la fotografía derecha muestra la recesión de una de las tormentas registradas. Es evidente el proceso de socavación y arrastre de material. En forma periódica se hace un seguimiento del avance de estas rocas a lo largo de la quebrada, indicando el diámetro y la evolución de su posición en el tiempo. Esta tarea se hace en forma simultánea con el registro de lluvia.

La evolución temporal del cauce será inferida de la topografía. Se levanto el eje longitudinal con el propósito de identificar secciones en la margen derecha e izquierda. En cada sección se indico el cambio de material para identificar las avalanchas registradas en el pasado. Estos puntos representan el nivel alcanzado por avalanchas anteriores, y se requieren para calibrar el modelo hidráulico, por lo que deben estar claramente indicados en cada sección.

\footnotetext{
${ }^{2}$ Lo abrupto de la topografía, el cambio constante de la sección transversal del cauce y la variabilidad de intensidad en las tormentas registradas obligaron a definir una estación de aforo con fácil acceso para el mantenimiento. Se identifico la sección de aguas abajo del puente como la de menor variabilidad. Allí se instalo una regleta.
} 

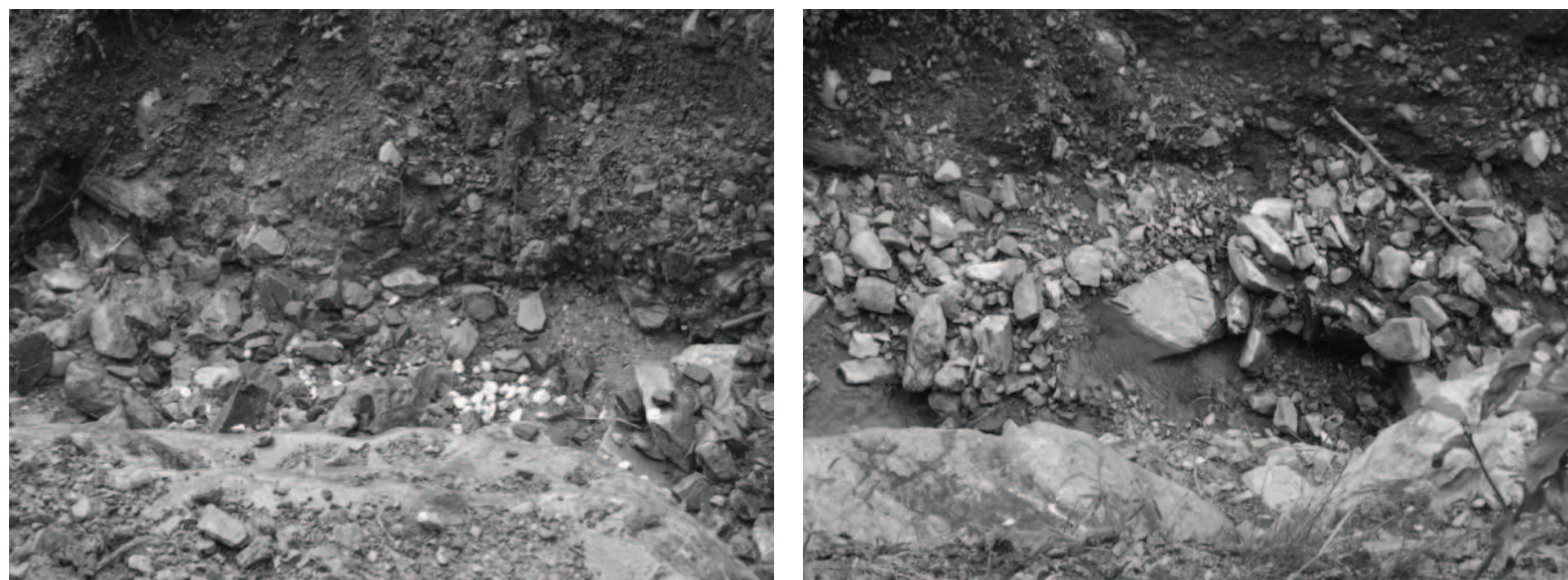

Figura 3. Sedimentos marcados para rastrear capacidad de transporte ${ }^{3}$

El paso vial actual ha demostrado ser insuficiente para el tránsito de avalanchas. En el año 2007 se realizó el diseño de un puente con un ancho de $11,40 \mathrm{~m}$ y una altura de $1,70 \mathrm{~m}$ (11). En la modelación hidráulica fue necesario suponer elementos adicionales que no fueron detallados en el diseño original, como fueron la pendiente y las condiciones hidráulicas en la entrada y salida de la estructura.

\section{Procesamiento de la información}

El análisis de la información se hizo en cada uno de los siguientes frentes:

- Estudio Hidrológico: Luego del estudio de la información disponible y los modelos lluvia escorrentía existentes, se seleccionó el Modelo del Soil Conservation Service, soportado por el software FLO2D. Para su implementación se requirió la construcción de tres archivos de datos: (1) Cuenca hidrográfica. (2) Modelo Hidrológico. (3) El tiempo de Simulación.

- Estudio Hidráulico: Luego de la revisión bibliográfica, se opto por resolver la ecuación de energía y continuidad dentro de un modelo distribuido. La teoría es aceptada por la Federal Emergency Management Agency (FEMA), quienes impulsan la implementación de herramientas como el software FLO 2D.

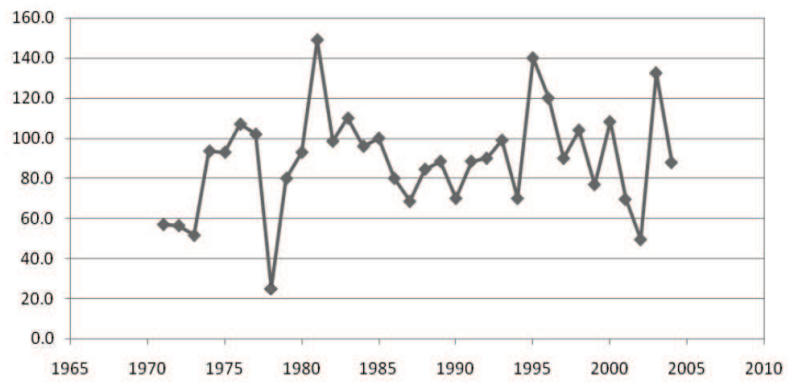

Como característica principal, FLO 2D tiene la habilidad para calcular el flujo en ocho (8) direcciones, las cuatro tradicionales más cuatro diagonales. De igual forma permite modelar obstrucciones al flujo como son las generadas por puentes, calles y edificaciones, además de considerar la variación espacial de la lluvia y la infiltración. Es un software desarrollado para trabajar en topografías abruptas. El modelo es alimentado con los resultados de los estudios hidrológicos, geológicos y geotécnicos.

\section{RESULTADOS Y DISCUSIÓN}

\section{Precipitación}

La estación El Túnel está a una altitud de 2480 msnm y posee una precipitación promedio anual 1977 mm. San Rafael. Está en la cota 1600 msnm y presenta una precipitación promedio anual 1725 mm. La Estación planadas está emplazada a 2350 msnm y registra una precipitación promedio anual $1824 \mathrm{~mm}$. Las estaciones inician registro en el año de 1971, aunque las estaciones El Túnel y San Rafael se suspenden en 1986. Puesto que el área de estudio es pequeña, al realizar el trazado de los polígonos de Thiessen se encuentra que la influencia de la estación Planadas es del $100 \%$, por ello será sólo se estudio éste registro.

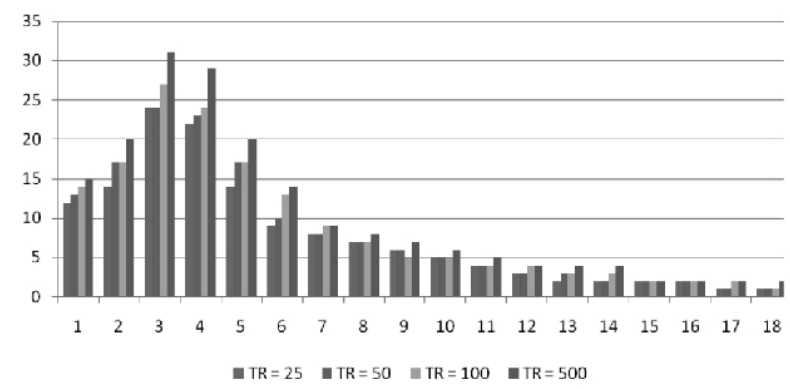

Figura 3 - Análisis de precipitación Estación Planadas (8)

${ }^{3}$ En la cuenca alta se pintaron rocas de diferente diámetro. En la fotografía izquierda se aprecia el fondo del cauce con rocas pintadas en diferentes colores y diámetros inferiores a los $10 \mathrm{~cm}$, con ausencia de flujo superficial. Durante el monitoreo se registró el desplazamiento de estas rocas. En la fotografía derecha se aprecia el transporte de sedimento para el mismo punto. 
Para el cálculo de caudales en modelos lluvia escorrentía, la principal suposición que se hace es considerar que los caudales máximos con periodos de retorno de 25, 50, 100 y 500 años son los generados por tormentas máximas con periodos de retorno, 25, 50, 100 y 500 años, respectivamente (12). Bajo esta premisa se procede entonces a calcular las lluvias máximas para estos periodos de retorno a partir de datos de lluvia diaria disponible en la estación Planadas. Las lecturas de precipitación, con registros diarios para varios años, fueron utilizadas para la estimación de precipitación máxima diaria. A partir de las lecturas diarias de la Estación Planadas se obtuvieron las precipitaciones máximas diarias para cada año (Imagen izquierda Figura 3).

Obtenidas las precipitaciones máximas diarias, se procedió a determinar los ajustes teóricos a las diferentes funciones de distribución de probabilidades, disponibles en la literatura, para luego compararlos con las series observadas. Del registro se descarta el valor de 25,0 mm, año 1978, donde sólo se registraron los meses de julio, septiembre, octubre, noviembre y diciembre. Para la definición de la profundidad de la tormenta de diseño se utilizó el software SMADA (13) verificando el comportamiento de la FDP Log Normal como un modelo teórico adecuado para representar el registro de la Estación Planadas (Figura 4). De los datos presentados en la imagen izquierda de la Figura 3 se observa que en el año de 1981 se registró una precipitación máxima en 24 horas de $149 \mathrm{~mm}$, valor cercano a un período de retorno de 50 años, hecho que se deduce al comparar éste con el resultado del modelo teórico (Figura 4). Luego, en 34 años de registro se presentó un evento con TR $=50$, por lo que en el futuro cercano es alta la probabilidad de que se presenten eventos con periodos de retorno mayores.

El hietograma de diseño (imagen derecha Figura 3) se construyó con la tormenta de primer cuartil con $50 \%$ de probabilidad desarrollado por Huff (14). Allí se observa una intensidad máxima de $144 \mathrm{~mm} / \mathrm{h}$ para $\mathrm{TR}=25$, valor que es superior al observado en el registro de la Estación Buenavista (108 mm/h). Con la duración seleccionada (tres horas), en la primera hora caen $95 \mathrm{~mm}$ para TR = 25 años, valor cercano al registrado en Buenavista.

\begin{tabular}{|c|c|c|c|c|c|c|c|}
\hline MISTRIB 2.13 - Distributio & lys & & & & & & $-\square x$ \\
\hline File Edit Statistics Calculators & & & & & & & \\
\hline Solout Diatribution & & Weibull & Data & Prediction & $\begin{array}{l}\text { Std. Dev. } \\
\text { St }\end{array}$ & $\Delta$ & \\
\hline Select Distrioution & 1 & 0.03 & 49.50 & 54.49 & 5.2823 & & Print \\
\hline Normal & 2 & 0.06 & $\overline{51.60}$ & 59.19 & 4.7872 & & \\
\hline U Normal & 3 & 0.09 & 56.40 & 62.49 & 4.4770 & & Help \\
\hline 0) 2 Parameter Lon Normal & 4 & 0.12 & 57.00 & 65.17 & 4.2533 & & Use \\
\hline & 5 & 0.15 & 68.50 & 67.49 & 4.0832 & & CTRL-INSERT \\
\hline 3 Parameter I on Normal & 6 & 0.18 & 69.60 & 69.59 & 3.9514 & & to copy \\
\hline $3 \mathrm{Par}$ ameter Log Normal & 7 & 0.21 & 70.00 & 71.53 & 3.8497 & & highlighted data \\
\hline Pearenn Tune III & 8 & 0.24 & 70.00 & 73.37 & 3.7731 & & $\begin{array}{l}\text { From } \\
\text { snreadsheet }\end{array}$ \\
\hline U rearson I gpe iII & 9 & 0.26 & 76.90 & 75.12 & 3.7185 & & SHIFT-INSERT \\
\hline O In Pearson Tune III & 10 & 0.29 & 80.00 & 76.81 & 3.6839 & & to paste data \\
\hline Log rearson I ype ill & 11 & 0.32 & 80.00 & 78.46 & 3.6678 & & into spreadsheet \\
\hline O Gumbel Tune I Futremal & 12 & 0.35 & 84.50 & 80.09 & 3.6691 & & \\
\hline Gumbel I gpe I Extremal & 13 & 0.38 & 87.90 & 81.69 & 3.6872 & & Mean \\
\hline & 14 & 0.41 & 88.50 & 83.29 & 3.7216 & - & $910515>>15$ \\
\hline & & \begin{tabular}{l|l|} 
Prob \\
\end{tabular} & R Period & Prediction & Std. Dev. & $\Delta$ & 91.0515 \\
\hline ter Log Normal & 1 & 0.998 & 500.0 & 183.40 & 16.4905 & & 2nd M \\
\hline${ }^{150} \mathrm{~J}$ & 2 & 0.990 & 100.0 & 159.37 & 12.9041 & & $548 \mathrm{en}$ \\
\hline & 3 & 0.980 & 50.0 & 148.69 & 11.3343 & & $5.548 \mathrm{e} U 2$ \\
\hline valu & 4 & 0.960 & 25.0 & 137.65 & 9.7388 & & Skew \\
\hline & 5 & 0.900 & 10.0 & 122.15 & 7.5780 & & DKE표 \\
\hline & 6 & 0.800 & 5.0 & 109.20 & 5.9098 & & \\
\hline $\begin{array}{llllll}8 & 1 & 1 & 1 & 1 & 1\end{array}$ & 7 & 0.667 & 3.0 & 98.38 & 4.7153 & & \\
\hline 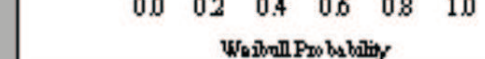 & 8 & 0.500 & 2.0 & 88.15 & 3.9203 & & \\
\hline 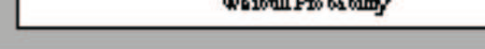 & 9 & & & & & $\boldsymbol{\nabla}$ & \\
\hline
\end{tabular}

Figura 4 - Resultados software SMADA para la estación Planadas (8) 


\section{Infiltración}

EI Soil Conservation Service (SCS) desarrolló un procedimiento para el análisis del fenómeno lluvia escorrentía. Este método goza de mucha popularidad en nuestro medio para determinar las tormentas de diseño, cuando se estudian caudales máximos. La información que sirve para sustentar este análisis se identificó en el recorrido de campo, por el cual se identifico que el uso actual y cobertura de suelo correspondía con un mosaico de pastos naturales, bosques nativos, rastrojo bajo y rastrojo alto, en porcentajes similares; los suelos corresponden con cenizas volcánicas que se pueden clasificar hidrológicamente en el Grupo B. Para la cuenca de la Qda. El Cofre, se estimo un número de curva de 62,57.

\section{Mapas de amenaza}

El análisis del fenómeno de remoción en masa no considero la estructura que en la actualidad existe en el paso vial hacía Quebradanegra. Considerando la tormenta con TR $=500$ años, a continuación se presentan los resultados de cuatro escenarios.

Escenario 1. Sólo considera la precipitación para un periodo de retorno de 500 años con el propósito de comprobar la respuesta del modelo a las observaciones realizadas en campo. Se construye un mapa de amenaza, mostrado en la imagen superior de la Figura 5, que indica un valor bajo de amenaza en inmediaciones al cruce vial y en un gran trayecto de la cuenca baja hasta la desembocadura con el Río Santo Domingo. Estos resultados están conformes con las observaciones realizadas en campo por los diferentes grupos de trabajo, y se correlacionan con la topografía del terreno.

Escenario 2. Al considerar un escenario con deslizamiento más la precipitación pero sin la estructura que se propone construir, las condiciones en la cuenca se tornan especiales en algunos puntos. Aunque la amenaza es baja en la mayor parte de la cuenca, ésta crece notablemente alrededor del caso urbano y en la cuenca alta, según se muestra la imagen inferior de la Figura 5. Se observa una zona de amenaza media en la parte alta de la cuenca, sector con mayor probabilidad de represamiento. Es necesario resaltar los aspectos negativos que se derivarían de la falla de uno de estos represamientos.

Escenario 3. Al considerar un escenario con precipitación y la construcción del puente que se tiene proyectado en el paso vial hacía Quebradanegra, se observa la deficiencia hidráulica de la estructura. El mapa de amenaza sigue mostrando condiciones bajas en la cuenca que se localizan en las mismas zonas que se identificaron el Escenario 1. Aparece un núcleo con amenaza baja y media en la parte superior del casco urbano del corregimiento de La Virginia (Figura 6). Está situación indica la deficiencia hidráulica del puente que se diseño en el 2007 y se pretende construir en el mediano plazo. 

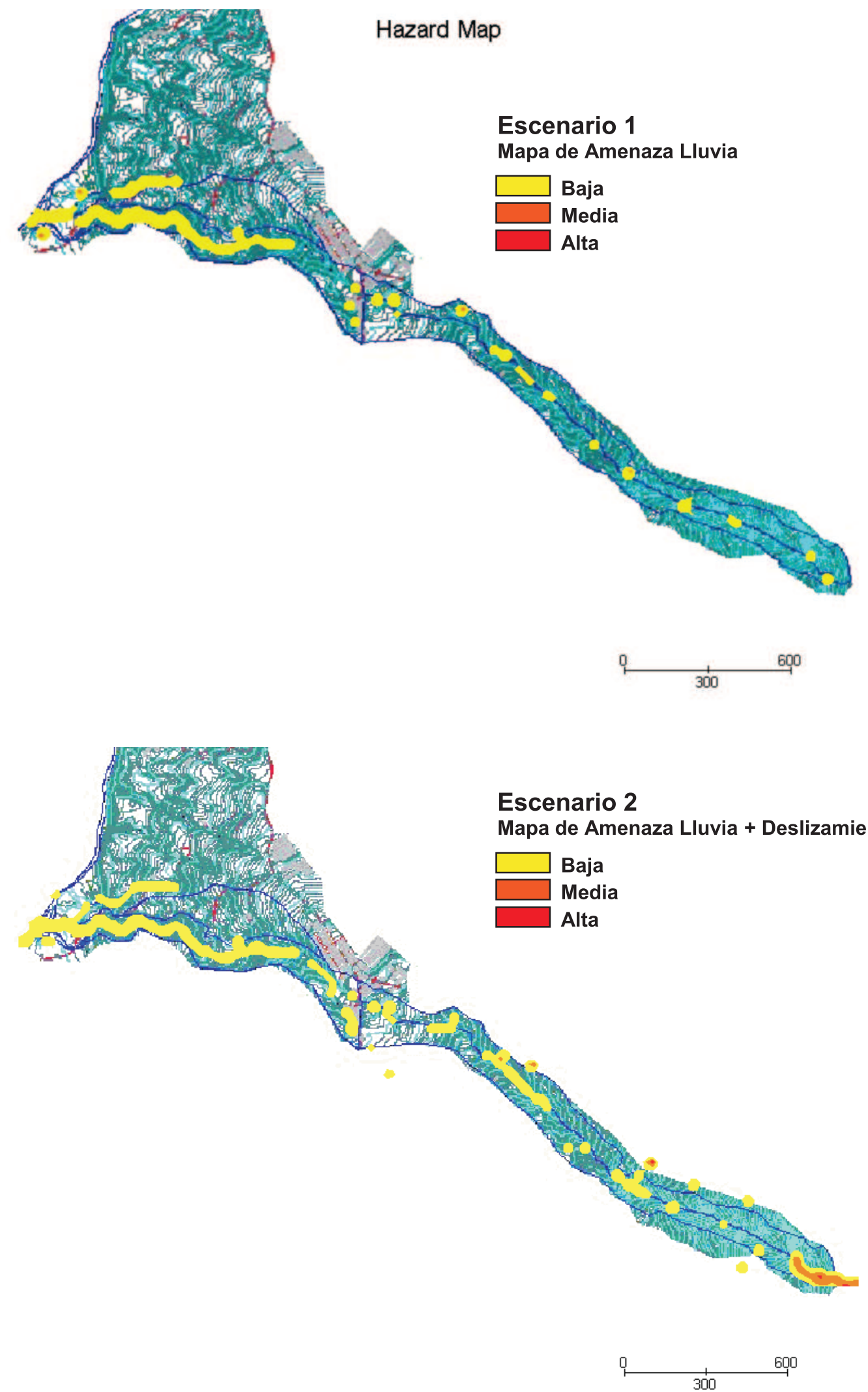

Figura 5 - Mapas de amenaza para escenarios 1 y 2 (8)

Rev. Invest. Univ. Quindío (20): 108 - 119. Armenia - Colombia 
García R., Pedro L., Monsalve D., Elkin A., Lozano S., Gabriel - 117 -
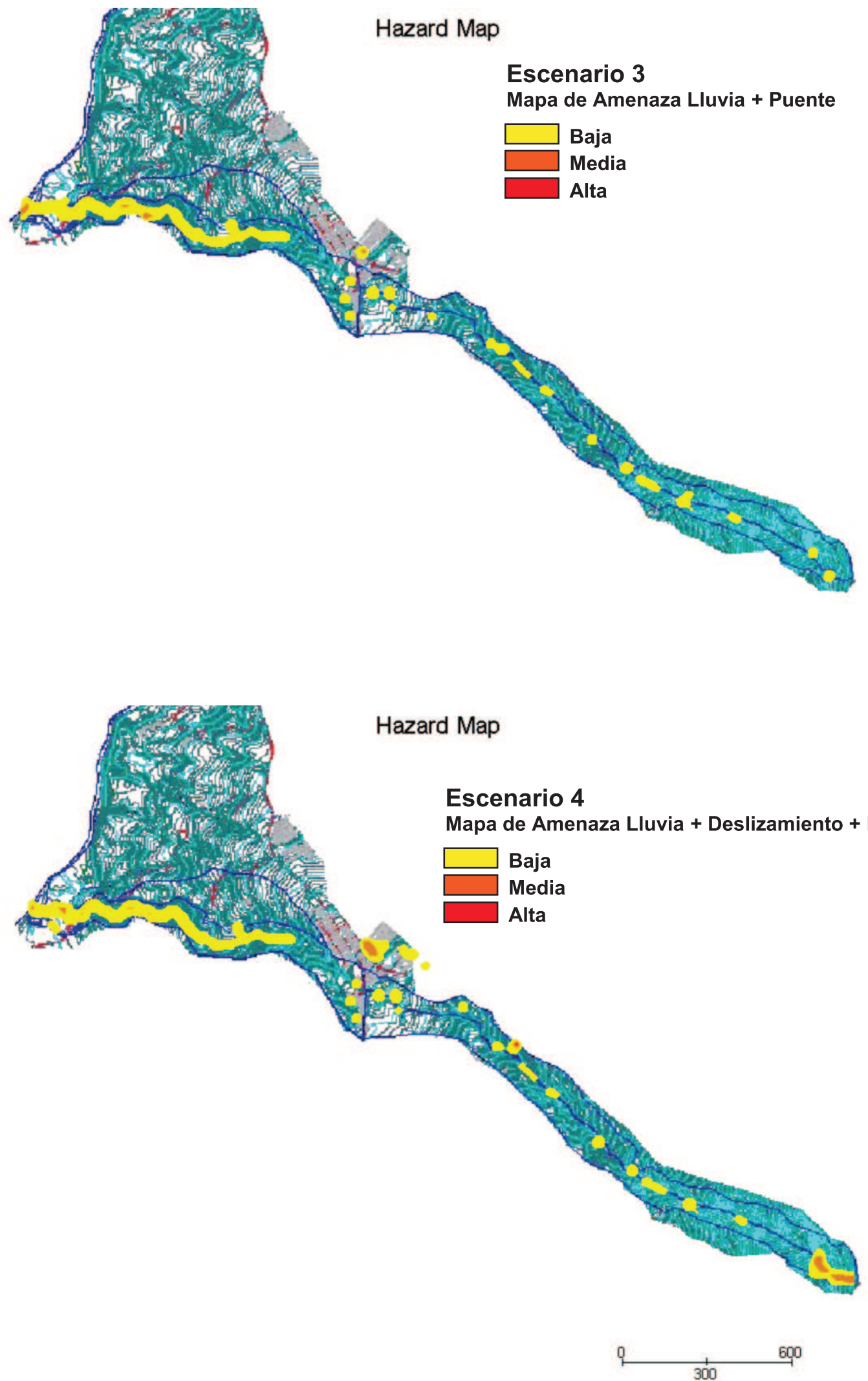

Figura 6 - Mapas de amenaza para escenarios 3 y 4 (8) 
Estudio de caso: amenaza por avalancha quebrada el cofre García R., Pedro L., Monsalve D., Elkin A., Lozano S., Gabriel Escenario 4. El más crítico de los escenario está representado por una tormenta con un período de retorno de 500 años que ocurra durante un intenso invierno, que con suelos saturados activaría deslizamientos latentes que corren hacía el puente. En este escenario se conservan las zonas descritas en el Escenario 2. Como hecho nuevo, aparece una zona de riesgo medio en el sector de San Luis y el núcleo en la parte alta del casco urbano se define como medio y se extiende hacia el colegio (Figura 6).

\section{CONCLUSIONES}

La Estación Planadas se encuentra localizada en una cota cercana al nivel superior de la Quebrada El Cofre. El registro de datos es continuo desde el año de 1971. En el registro una precipitación máxima en 24 horas con una profundidad de $149 \mathrm{~mm}$, presentada en marzo de 1981. De acuerdo a la FDP Log Normal tiene un periodo de retorno de 50 años. Este valor se presenta en una ventana de tiempo que está próxima a alcanzar los 40 años. No existe registro en la memoria de los pobladores de inundaciones o avalanchas generadas por esta precipitación en el año de 1981. Para la época, la cuenca se encontraba en mejores condiciones de cobertura natural y sin el cambio estructural que pudo haber inducido el terremoto de 1999. Como hecho curioso, esta precipitación se presenta después del terremoto de 1979.

Hoy la cuenca presenta laderas inestables, cambios de cobertura, reducción de las tasas de infiltración y almacenamiento de sedimentos en el cauce. Desde la inferencia estadística, es probable que se presenten tormentas con periodos de retorno superiores a los cincuenta años en el corto o mediano plazo. Durante el trabajo de se observó un cauce móvil y activo que arrastra material grueso y que durante grandes crecientes es capaz de movilizar grandes bloques de roca. La caída de una gran tormenta podría provocar el represamiento por la falla de las zonas inestables, la remoción del material acumulado en el lecho y la generación de un flujo de escombros.

Los resultados de la simulación orientan la atención hacia dos zonas que no fueron objeto directo del estudio. La Quebrada Espartillal que pasa por el extremo sur del corregimiento de La Virginia, podría generar eventos similares. Aunque en la actualidad no se presenta flujo de escombros, el sólo flujo de agua representa una amenaza media para los habitantes del sector. Otra zona que demanda especial atención está representada por la cuenca baja, donde existen conflictos con el uso del suelo. Son pendientes pronunciadas que se encuentran con cultivos semestrales y suelos aluviales que se encuentran en zona de pastoreo.

Bajo estas condiciones es necesario realizar actividades para mitigar el impacto generado por cualquiera de los escenarios analizados. Son varias las acciones que se pueden emprender. A continuación se describen,

1. Mejorar la capacidad de auto-reguladora de la cuenca. A través de una campaña de reforestación con flora nativa se deberían sustituir todos aquellos cultivos que entren en conflicto con el uso del suelo. Es necesario adelantar un estudio detallado que identifique las áreas de reforestación.

2. Remover el material que se encuentra depositado en el lecho. El propósito es generar volúmenes de almacenamiento para el material que será transportado por un gran evento. Es necesario implementar y programar actividades periódicas de remoción de sedimentos. Al finalizar cada temporada invernal, el objeto será la remoción de los grandes bloques depositados a lo largo del cauce y el dragado de las zonas de menor pendiente, como la Curva de San Luis.

3. Remover el material de las zonas inestables. Desde las laderas se arrastra material que contribuye al incremento de la densidad y la viscosidad del flujo, especialmente durante una creciente. Bajo ciertas condiciones se podría represar el cauce. Si estas zonas se estabilizan con una técnica que incluya la remoción del material suelto, se logrará reducir los factores detonantes de una avalancha.

4. Demoler el puente en el paso vial. La estructura que en la actualidad se encuentra en condiciones físicas regulares, representa una constricción al flujo, como se hizo evidente en el evento de febrero de 2008. La demolición de la estructura es condición necesaria para mejorar el transito del agua por su cauce natural. En su lugar, se puede habilitar un badén, que además de facilitar el tránsito vehicular hacía y desde Quebradanegra, no representa un obstáculo para el flujo natural de las aguas drenadas por la cuenca hidrográfica de la Quebrada EI Cofre.

5. Evaluar un nuevo paso vial. Considerando los resultados arrojados por los Escenarios 2 y 4 , se concluye que las condiciones hidráulicas del puente que se desea construir (11) no son apropiadas para el flujo de escombros que podría presentarse en el cauce. Las condiciones simuladas son indicadoras de la necesidad de proyectar obras que no obstruyan el flujo natural de las aguas de escorrentía. Una solución a largo plazo exige el diseño de un paso vial cuyos cimientos no se encuentren en áreas aledañas al cauce de la quebrada El Cofre. 


\section{BIBLIOGRAFÍA}

1 Aguilar, V. Mendoza, D. 2002. Aproximación a un modelo de susceptibilidad a movimientos de masa en el eje cafetero, Colombia. Trabajo de grado. Universidad del Valle. Calí.

2 Rojas, G. 1999. La Virginia, un Armero chiquito. En: La Crónica del Quindío. Diciembre 28 de 1999. Págs. 2A a 3A.

3 Rojas, G. 1999. La Virginia, en alerta roja. En: La Crónica del Quindío. Diciembre 28 de 1999. Págs. 3A a 4A.

4 Corporación Autónoma Regional del Quindío. CRQ. 2006. Informe de gestión y resultados. Primer Semestre de 2006. Plan de Acción Trienal 2004-2006. Armenia.

5 Nuestra Tele Noticias. (2008, Febrero 22). Alerta roja en corregimiento de Calarcá por invierno. Consultado Julio $21,2009$. En: http://www.ntn24.com/content/alerta-roja-corregimiento-calarca-invierno

6 Sitio oficial de Calarcá en Quindío, Colombia. (2009, Julio 24). Consultado Julio 24, 2009. En:

http://calarca-quindio.gov.co/nuestromunicipio.shtml?apc=m1l1--\&m=f\#geografia

7 Sitio comercial Calarcá (2009, Julio 24). Consultado Julio 24, 2009. En: http://www.calarca.net/geografia.html

8 García R., P. L. 2009. Estudio de caso Quebrada el cofre Fase II. Volumen III-Estudio de Hidrología. Centro de Investigación, Desarrollo y Estudio del Recurso Hídrico y el Ambiente, CIDERA. Universidad del Quindío. Armenia.

9 Toro B, C. 2009.Curvas sintéticas de Intensidad Duración Frecuencia para el departamento del Quindío. Centro de Investigación, Desarrollo y Estudio del Recurso Hídrico y el Ambiente, CIDERA. Universidad del Quindío. Armenia.

10 Arango C, S.E. 2003. Boletín Meteorológico-2002. Corporación Autónoma Regional del Quindío. Universidad del Quindío. Facultad de Ingeniería. Armenia.

11Fontal, C.A. ING. 2007. Mejoramiento vial mediante construcción de puente vehicular sobre la Quebrada El Cofre. Corregimiento La Virginia. Calarcá. Secretaría de Planeación Municipal. Calarcá.

12 IDEA, 2006. Estudio hidrológico e hidráulico de la cuenca del río San Eugenio para generar una información básica para la intervención de la cuenca en su tramo urbano. Fase 1. Universidad Nacional de Colombia, Instituto de Estudios Ambientales.

13 Wanielista, M.P. Kersten, R. Eaglin, R.D. 1997. Hydrology: Water Quantity and Quality Control. Second Edition. John Wiley and Sons. Stormwater Management and Design Aid. SMADA 6.43. Statiscal Distribution Analysis. Distrib 2.13. Florida.

14Chow, V. T., et al. 1994. Hidrología Aplicada. Mc GRAW-HILL. Santafé de Bogotá. 